\title{
Typical gastric mucosa with ulcer found by endoscopy in Meckel's diverticulum
}

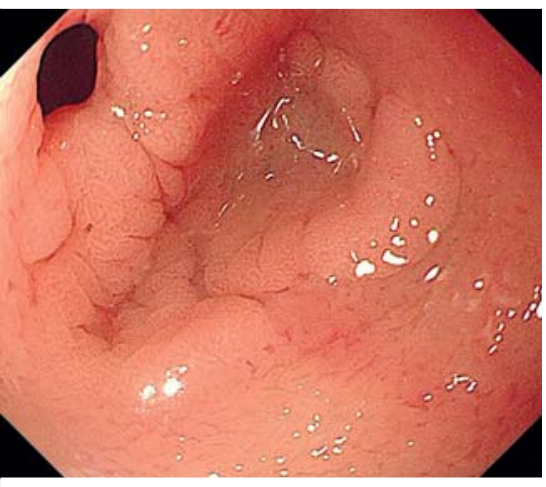

Fig. 1 Endoscopic appearance of a diverticulum in a 19-year-old male under white light.

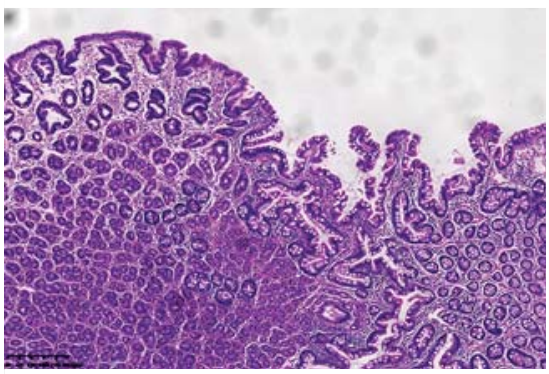

Fig. 4 Pathological analysis: transition between the ectopic gastric mucosa and the intestinal mucosa revealed by hematoxylin and eosin staining.

A 19-year-old male experienced recurrent rectal bleeding and syncope twice within a month. Laboratory examination showed iron-deficiency anemia with a low hemoglobin level of $78 \mathrm{~g} / \mathrm{L}$. Gastrointestinal endoscopy revealed only a tiny ulcer in the gastric antrum $(0.3 \mathrm{~cm}$ in diameter, healing period), and no abnormality was observed in the large intestine. Transanal single-balloon enteroscopy revealed a bifurcation of the intestinal tract $100 \mathrm{~cm}$ from the ileocecal valve, and a diagnosis of Meckel's diverticulum with ulcer $(0.5 \mathrm{~cm}$ in diameter, active period) was considered ( $\triangleright$ Fig. 1 ). The boundary between the small intestinal villi and gastric mucosa was clearly observed on narrow-band imaging and after indigo carmine staining ( $\mathbf{F i g . 2}$,

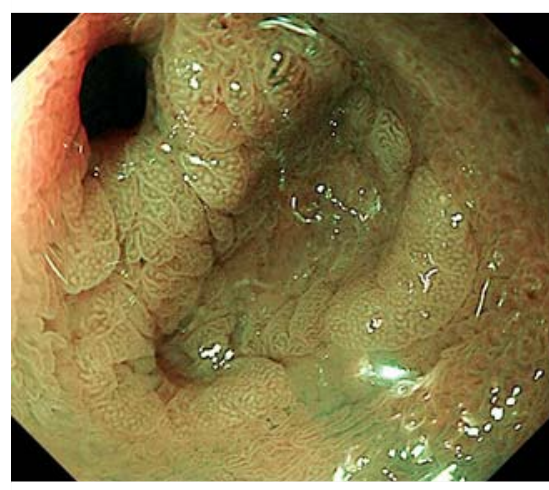

Fig. 2 Appearance of the diverticulum under narrow-band imaging.

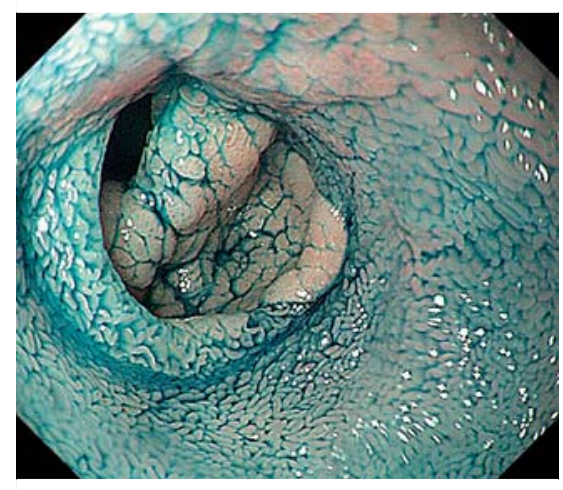

> Fig. 3 Appearance of the diverticulum after indigo carmine staining.

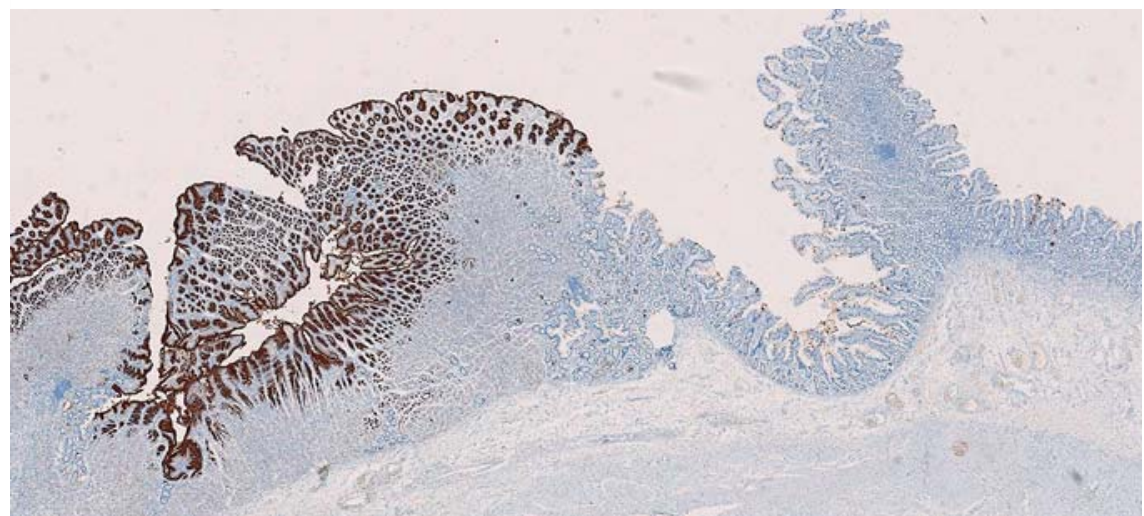

Fig.5 Pathological analysis: MUC5AC antibody reaction with the gastric epithelium.

- Fig. 3; Video 1). We diagnosed the patient with ectopic gastric mucosa with ulcer in Meckel's diverticulum on the basis of the endoscopic manifestation; in previous reports this diagnosis relied on pathological analysis rather than endoscopic observation. The patient subsequently underwent surgery, and pathological analysis (including MUC5AC antibody reaction) confirmed the presence of ectopic gastric mucosa with ulcer ( $\triangleright$ Fig. 4, $>$ Fig.5). The patient has since been asymptomatic on follow-up.

Diverticula can cause complications such as gastrointestinal bleeding [1]; complications occur mostly in children, rarely in adults [2,3]. Bleeding from Meckel's diverticulum due to ectopic gastric mu- cosa is the most common clinical presentation, particularly in young patients [4]. Tips: Ectopic gastric mucosa can be better observed by endoscopic staining. Rigid thinking should be avoided to allow other considerations when the level of bleeding is not consistent with a gastric ulcer. Age is not an absolute criterion of disease identity; other factors matter as well.

Endoscopy_UCTN_Code_CCL_1AC_2AF

\section{Competing interests}

The authors declare that they have no conflict of interest. 


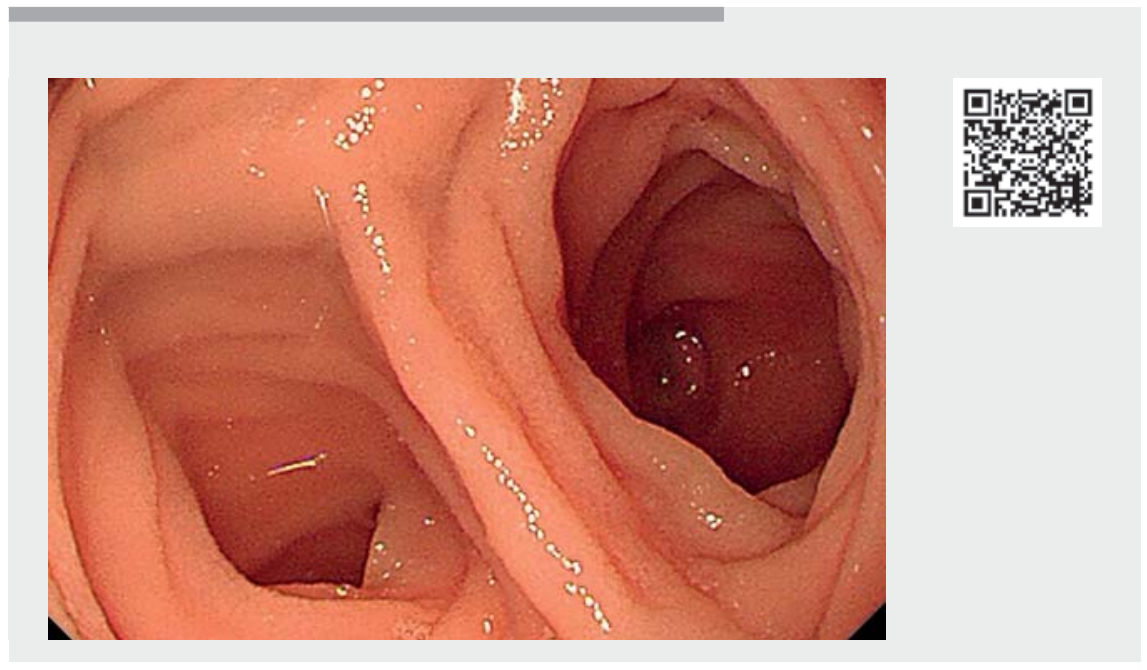

Video 1 Gastric mucosa with ulcer found by endoscopy in Meckel's diverticulum. Single-balloon enteroscopy shows two lumens. The boundary between the small intestinal villi and gastric mucosa was clearly observed on narrow-band imaging and after indigo carmine staining.
Bibliography

Endoscopy 2022; 54: E154-E155

DOI $10.1055 / a-1471-1857$

ISSN $0013-726 \mathrm{X}$

published online 28.4.2021

(C) 2021. Thieme. All rights reserved.

Georg Thieme Verlag KG, Rüdigerstraße 14, 70469 Stuttgart, Germany

\section{ENDOSCOPY E-VIDEOS}

https://eref.thieme.de/e-videos

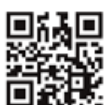

Endoscopy E-Videos is an open access online section, reporting on interesting cases and new techniques in gastroenterological endoscopy. All papers include a high quality video and all contributions are freely accessible online. Processing charges apply (currently EUR 375), discounts and wavers acc. to HINARI are available.

This section has its own submission website at

https://mc.manuscriptcentral.com/e-videos
Hong Zhang ${ }^{1,2}$, Zhen Yang ${ }^{2}$, Yan-Hong Wang ${ }^{2}$ Shi-Ming Yang ${ }^{2}$, Lu Liu' ${ }^{2}$, Jian-Ying Bai ${ }^{2}$, ChaoQiang Fan ${ }^{2}$

1 Department of Gastroenterology, Affiliated Hospital of North Sichuan Medical College, Nanchong, Sichuan, China

2 Department of Gastroenterology, Xinqiao Hospital, the Second Affiliated Hospital of Army Medical University, Chongqing, China

\section{Corresponding author}

\section{Chao-Qiang Fan, MD}

Department of Gastroenterology, Xinqiao Hospital, Second Affiliated Hospital of Army Medical University, 83 Xinqiao Street, Shapingba District, Chongqing 400037, P.R. China

fcqxhkwjs@126.com

\section{References}

[1] Rangan V, Lamont JT. Small bowel diverticulosis: pathogenesis, clinical management, and new concepts. Curr Gastroenterol Rep 2020; 22: 4

[2] Kuru S, Kismet K. Meckel's diverticulum: clinical features, diagnosis and management. Rev Esp Enferm Dig 2018; 110: 726 732

[3] Lequet J, Menahem B, Alves A et al. Meckel's diverticulum in the adult. J Visc Surg 2017; 154: 253-259

[4] Sagar J, Kumar V, Shah DK. Meckel's diverticulum: a systematic review. J R Soc Med 2006; 99: 501-505 